\title{
INVERSE PROBLEM TO DETERMINE DEGENERATE MEMORY KERNELS IN HEAT FLUX WITH THIRD KIND BOUNDARY CONDITIONS
}

\author{
E. PAIS $^{1}$ and J. JANNO ${ }^{2}$ \\ ${ }^{1}$ Institute of Mathematics at Tallinn UT, \\ Ehitajate rd 5, 19086 Tallinn, Estonia \\ ${ }^{2}$ Institute of Cybernetics at Tallinn UT \\ Akadeemia rd 21, 12618, Tallinn, Estonia \\ E-mail: ennopais@stv.ee; janno@ioc.ee \\ Received May 22, 2005; revised September 24, 2006; published online December 15, 2006
}

\begin{abstract}
An inverse problem to determine degenerate time- and space-dependent relaxation kernels of internal energy and heat flux with third kind boundary conditions by means of temperature measurements is considered. Existence and uniqueness of a solution to the inverse problem are proved.
\end{abstract}

Key words: inverse problem, memory kernel, heat flow

\section{Introduction}

Constitutive relations in the theory of heat conduction in materials with thermal memory contain time-dependent (and in the case of non-homogeneity also space-dependent) memory (or relaxation) kernels $[1,9,10,13]$. These kernels are often unknown in the practice. To determine them, inverse problems are used. Several papers discuss the identification of space- and time-dependent kernels in continuous non-homogeneous bodies. They represent different approaches and apply different methods to study the inverse problems, e.g. in $[2,5]$ stratified bodies are considered, [4] studies the identification of a kernel by means of a restricted Dirichlet-to-Neumann map.

In some context the kernels can be degenerate, i.e. representable as finite sums of products of known space-dependent functions times unknown time-dependent coefficients. This is so when either the medium is piecewise continuous or a problem for a general kernel is replaced by a related problem for an approximated kernel. The unknown coefficients are recovered by 
a finite number of measurements of certain time-dependent characteristics of the solution of the direct problem. In $[6,7,8]$ inverse problems of such a type were studied. In particular, [8] treats a problem with boundary conditions of the third kind. The mentioned papers are limited to the simplified case when the model contains only the relaxation kernel of heat flux. However, a more precise model of a material with thermal memory involves two relaxation kernels contained in basic constitutive relations: kernels of internal energy and heat flux.

In the present paper we study an inverse problem to determine degenerate nonhomogeneous relaxation kernels of internal energy and heat flux by means of a finite number of measurements of temperature in fixed points over the time. The corresponding heat conduction model contains boundary conditions of the third kind. The corresponding case of boundary conditions of the first kind (that is less relevant from the physical point of view) was treated in [11]. Third kind boundary conditions bring along certain complications related to the non-vanishing Green function on the boundary. In [8] these problems were overcome assuming certain vanishing conditions at the boundary either for the derivative of the initial condition or for the memory kernel. In this paper we present an approach that doesn't assume such vanishing conditions.

In Section 2 we formulate the direct and inverse problems and in Section 3 apply the Laplace transform to them. In Section 4 we rewrite the transformed problems in the fixed-point form. Sections 5 and 6 contain some auxiliary results for the direct problem. Main existence and uniqueness results for the inverse problem are included in Section 7 of the paper.

\section{Formulation of Problem}

We consider the heat flow in a rigid non-homogeneous bar consisting of a material with thermal memory. For a sake of simplicity we assume the rod to be of the unit length. Then, in the linear approximation we obtain the following heat equation

$$
\begin{array}{r}
\beta(x) \frac{\partial}{\partial t} u(x, t)+\frac{\partial}{\partial t} \int_{0}^{t} n(x, t-\tau) u(x, \tau) d \tau=\frac{\partial}{\partial x}\left(\lambda(x) u_{x}(x, t)\right) \\
-\frac{\partial}{\partial x} \int_{0}^{t} m(x, t-\tau) u_{x}(x, \tau) d \tau+r(x, t), \quad x \in(0,1), t>0,
\end{array}
$$

where $x \in(0,1)$ is the space coordinate and $t \in \mathbb{R}$ is the time. Here $u$ is the temperature of the bar, which is assumed to be zero for $t<0, e$ is the internal energy and $r$ is the heat supply. Moreover, $\beta$ and $\lambda$ stand for the heat capacity and the heat conduction coefficient, respectively. The model contains two memory kernels $n$ and $m$, being the relaxation kernels of the integral energy and the heat flux, respectively.

The function $u(x, t)$ is assumed to satisfy the initial conditions 


$$
u(x, 0)=\varphi(x), \quad x \in(0,1)
$$

and the boundary conditions of the third kind

$$
-q(0, t)=\alpha_{1}\left(u(0, t)-f_{1}(t)\right), \quad q(1, t)=\alpha_{2}\left(u(1, t)-f_{2}(t)\right), \quad t>0,
$$

with given functions $\varphi$ on $[0,1]$ and $f_{j}, j=1,2$ on $[0, \infty)$. Here $q(x, t)$ is a heat flux given by

$$
q(x, t)=-\lambda(x) u_{x}(x, t)+\int_{0}^{t} m(x, t-\tau) u_{x}(x, \tau) d \tau .
$$

In view of this equality the boundary conditions can be written as

$$
\begin{aligned}
\lambda(0) u_{x}(0, t)-\int_{0}^{t} m(0, t-\tau) u_{x}(0, \tau) d \tau & =\alpha_{1}\left(u(0, t)-f_{1}(t)\right), \\
-\lambda(1) u_{x}(1, t)+\int_{0}^{t} m(1, t-\tau) u_{x}(1, \tau) d \tau & =\alpha_{2}\left(u(1, t)-f_{2}(t)\right) .
\end{aligned}
$$

Equation (2.1) with the conditions (2.2) and (2.3) form the direct problem for the temperature $u$.

In an inverse problem we seek for the kernels $n$ and $m$. We restrict ourselves to the case of the kernels in the following degenerate forms

$$
n(x, t)=\sum_{j=1}^{N_{1}} \nu_{j}(x) n_{j}(t), \quad m(x, t)=\sum_{k=1}^{N_{2}} \mu_{k}(x) m_{k}(t),
$$

where $\nu_{j}, j=1, \ldots, N_{1}, \mu_{k}, k=1, \ldots, N_{2}$ are given $x$-dependent functions and $n_{j}, j=1, \ldots, N_{1}, m_{k}, k=1, \ldots, N_{2}$ are unknown time-dependent coefficients. Formulas (2.4) hold, for instance, when the medium is piecewise continuous, where $n_{j}$ and $m_{k}$ are characteristic functions or smooth approximations of characteristic functions of the subdomains of homogeneity. In general case (2.4) can be interpreted as finite-dimensional approximations of the actual kernels.

We are going to recover the unknowns $n_{j}$ and $m_{k}$ by the measurement of the temperature in $N=N_{1}+N_{2}$ different interior points $x_{i} \in(0,1)$, $i=1, \ldots, N$, i.e., by the additional conditions

$$
u\left(x_{i}, t\right)=h_{i}(t), \quad i=1, \ldots, N, \quad t>0,
$$

where $h_{i}$ are given functions. Summing up, the relations (2.1)-(2.5) form the inverse problem for $n$ and $m$.

\section{Application of the Laplace Transform}

Applying the Laplace transform to equation (2.1) with initial condition (2.2) and taking in consideration (2.4) we obtain 


$$
\begin{aligned}
\beta(x)[p U(x, p)-\varphi(x)]+p \sum_{j=1}^{N_{1}} N_{j}(p) \nu_{j}(x) U(x, p) \\
=\frac{\partial}{\partial x}\left(\lambda(x) U_{x}(x, p)\right)-\sum_{k=1}^{N_{2}} M_{k}(p) \frac{\partial}{\partial x}\left(\mu_{k}(x) U_{x}(x, p)\right)+R(x, p),
\end{aligned}
$$

where $N_{j}=\mathcal{L} n_{j}, M_{k}=\mathcal{L} m_{k}, R=\mathcal{L} r$,

$$
U(x, p)=\mathcal{L} u(x, t)=\int_{0}^{\infty} e^{-p t} u(x, t) d t .
$$

The boundary conditions (2.3) are transformed to

$$
\begin{gathered}
\lambda(0) U_{x}(0, p)=\alpha_{1}\left[U(0, p)-F_{1}(p)\right]+\sum_{k=1}^{N_{2}} \mu_{k}(0) M_{k}(p) U_{x}(0, p), \\
-\lambda(1) U_{x}(1, p)=\alpha_{2}\left[U(1, p)-F_{2}(p)\right]-\sum_{k=1}^{N_{2}} \mu_{k}(1) M_{k}(p) U_{x}(1, p),
\end{gathered}
$$

where $F_{j}=\mathcal{L} f_{j}, j=1,2$. The additional conditions (2.5) get the form

$$
U\left(x_{i}, p\right)=H_{i}(p), \quad i=1, \ldots, N=N_{1}+N_{2}
$$

with $H_{i}(p)=\mathcal{L} h_{i}$.

The goal of this section is to rewrite the problem for $U$ in a form of a system of integral equations. To this end we introduce the differential operator

$$
(L U)(x, p)=\frac{\partial}{\partial x}\left(\lambda(x) U_{x}(x, p)\right)-\beta(x) p U(x, p), \quad x \in(0,1) .
$$

and represent equation (2.1) in the form

$$
\begin{aligned}
(L U)(x, p) & =p \sum_{j=1}^{N_{1}} N_{j}(p) \nu_{j}(x) U(x, p) \\
& +\sum_{k=1}^{N_{2}} M_{k}(p) \frac{\partial}{\partial x}\left(\mu_{k}(x) U_{x}(x, p)\right)-R(x, p)-\beta(x) \varphi(x) .
\end{aligned}
$$

Let us denote by $G(x, y, p)$ the Green function of operator $L$ with homogeneous third kind boundary conditions, i.e.

$$
\left\{\begin{array}{l}
L_{y} G(x, y, p)=\delta(x, y), x \in(0,1), y \in(0,1), \\
\lambda(0) G_{y}(x, 1, p)=\alpha_{1} G(x, 0, p),-\lambda(1) G_{y}(x, 0, p)=\alpha_{2} G(x, 1, p), x \in(0,1) .
\end{array}\right.
$$

Then the solution of (3.3) is given by

$$
\begin{aligned}
U(x, p) & =p \sum_{j=1}^{N_{1}} N_{j}(p) \int_{0}^{1} G(x, y, p) \nu_{j}(y) U(y, p) d y \\
& +\sum_{k=1}^{N_{2}} M_{k}(p) \int_{0}^{1} G(x, y, p) \frac{\partial}{\partial y}\left(\mu_{k}(y) U_{y}(y, p)\right) d y-F(x, p),
\end{aligned}
$$


where

$$
\begin{aligned}
F(x, p) & =\int_{0}^{1} G(x, y, p)[\beta(y) \varphi(y)+R(y, p)] d y+G(x, 1, p) \\
& \times\left[\lambda(1) U_{x}(1, p)+\alpha_{2} U(1, p)\right]-G(x, 0, p)\left[\lambda(0) U_{x}(0, p)-\alpha_{1} U(0, p)\right] .
\end{aligned}
$$

Due to (3.1) the latter formula can be rewritten in the form

$$
\begin{aligned}
F(x, p)= & \int_{0}^{1} G(x, y, p)[\beta(y) \varphi(y)+R(y, p)] d y \\
& +\left(\alpha_{1} F_{1}(p)-\sum_{k=1}^{N_{2}} \mu_{k}(0) M_{k}(p) U_{x}(0, p)\right) G(0, x, p) \\
& +\left(\alpha_{2} F_{2}(p)+\sum_{k=1}^{N_{2}} \mu_{k}(1) M_{k}(p) U_{x}(1, p)\right) G(1, x, p) .
\end{aligned}
$$

Integrating the integrals in the second sum of (3.4) by parts and using the symmetry relations $G(x, 1, p)=G(1, x, p), G(x, 0, p)=G(0, x, p)$, we obtain

$$
\begin{aligned}
U(x, p)= & \sum_{j=1}^{N_{1}} p N_{j}(p) \int_{0}^{1} G(x, y, p) \nu_{j}(y) U(y, p) d y \\
& -\sum_{k=1}^{N_{2}} M_{k}(p) \int_{0}^{1} G_{y}(x, y, p) \mu_{k}(y) U_{y}(y, p) d y-Q(x, p),
\end{aligned}
$$

where

$$
\begin{aligned}
Q(x, p)= & \int_{0}^{1} G(x, y, p)[\beta(y) \varphi(y)+R(y, p)] d y \\
& +\alpha_{1} F_{1}(p) G(0, x, p)+\alpha_{2} F_{2}(p) G(1, x, p) .
\end{aligned}
$$

Further, differentiating (3.4) with respect to $x$ we obtain the equation for $U_{x}(x, p)$

$$
\begin{aligned}
U_{x}(x, p) & =\sum_{j=1}^{N_{1}} p N_{j}(p) \int_{0}^{1} G_{x}(x, y, p) \nu_{j}(y) U(y, p) d y \\
& +\sum_{k=1}^{N_{2}} M_{k}(p) \int_{0}^{1} G_{x}(x, y, p) \frac{\partial}{\partial y}\left(\mu_{k}(y) U_{y}(y, p)\right) d y-F_{x}(x, p) .
\end{aligned}
$$

We split the second integral in (3.8) into two parts, from 0 to $x$ and from $x$ to 1 , and integrate them by parts. Taking into consideration the jump relation (see [12]) 


$$
G_{x}(x, x-0, p)-G_{x}(x, x+0, p)=\frac{1}{\lambda(x)}, \quad 0<x<1
$$

we get

$$
\begin{aligned}
U_{x}(x, p) & =\frac{1}{\lambda(x)} \sum_{k=1}^{N_{2}} M_{k}(p) \mu_{k}(x) U_{x}(x, p)+\sum_{j=1}^{N_{1}} p N_{j}(p) \int_{0}^{1} G_{x}(x, y, p) \\
& \times \nu_{j}(y) U(y, p) d y-\sum_{k=1}^{N_{2}} M_{k}(p)\left(\int _ { 0 } ^ { 1 } \left(G_{x y}(x, y, p) \mu_{k}(y) U_{y}(y, p) d y\right.\right. \\
& \left.-\mu_{k}(1) U_{x}(1, p) G_{x}(x, 1, p)+\mu_{k}(0) U_{x}(0, p) G_{x}(x, 0, p)\right)-F_{x}(x, p) .
\end{aligned}
$$

Thus, by (3.5) and (3.7) we have the following equation for $U_{x}(x, p)$ :

$$
\begin{aligned}
U_{x}(x, p)= & \frac{1}{\lambda(x)} \sum_{k=1}^{N_{2}} M_{k}(p) \mu_{k}(x) U_{x}(x, p) \\
& +\sum_{j=1}^{N_{1}} p N_{j}(p) \int_{0}^{1} G_{x}(x, y, p) \nu_{j}(y) U(y, p) d y \\
& -\sum_{k=1}^{N_{2}} M_{k}(p) \int_{0}^{1} G_{x y}(x, y, p) \mu_{k}(y) U_{y}(y, p) d y-Q_{x}(x, p)
\end{aligned}
$$

with $Q$ given by (3.7). Summing up,(3.6) and (3.9) form a system of integral equations for functions $U(x, p)$ and $U_{x}(x, p)$.

\section{Fixed-Point Systems for Inverse and Direct Problems}

In this section we deduce a fixed-point system for the inverse problem in the Laplace domain and transform further the system for $U$ and $U_{x}$. From equation (3.6) and (3.2) we obtain

$$
\begin{aligned}
\sum_{j=1}^{N_{1}} p N_{j}(p) & \int_{0}^{1} p G\left(x_{i}, y, p\right) \nu_{j}(y) p U(y, p) d y-\sum_{k=1}^{N_{2}} \sqrt{p} M_{k}(p) \sqrt{p} \\
& \times \int_{0}^{1} G_{y}\left(x_{i}, y, p\right) \mu_{k}(y) p U_{y}(y, p) d y=p^{2}\left[H_{i}(p)+Q\left(x_{i}, p\right)\right]
\end{aligned}
$$

for $i=1, \ldots, N$, where $Q$, given by (3.7), depends only on the data of the problem.

Firstly, let us study the behaviour of this equation in the process $\operatorname{Re} p \rightarrow$ $\infty$. Suppose a priori that the inverse problem has a solution $n_{k}, m_{l}$ with the following properties 
1. $n_{k}$ are differentiable, implying $p N_{k}(p) \rightarrow n_{k}(0)$ as $\operatorname{Re} p \rightarrow \infty$;

2. $\sqrt{p} M_{l}(p) \rightarrow 0$ as $\operatorname{Re} p \rightarrow \infty$;

3. A solution of the direct problem $u$ corresponding to these $n_{k}, m_{l}$ satisfies the relation

$$
\frac{\partial}{\partial t} u(\cdot, t) \in C^{1}[0,1], \quad t>0 .
$$

Then from item 3 and initial condition (2.1) we have

$$
p U(x, p) \rightarrow \varphi(x), \quad p U_{x}(x, p) \rightarrow \varphi^{\prime}(x) \text { as } \operatorname{Re} p \rightarrow \infty .
$$

Using these asymptotic relations, the items 1, 2 above and assertions (5.5) and (5.8) of Lemmas 1 and 2 below we obtain from (4.1) the equalities

$$
-\sum_{k=1}^{N_{1}} n_{k}(0) \frac{1}{\beta\left(x_{i}\right)} \nu_{k}\left(x_{i}\right) \varphi\left(x_{i}\right)=\lim _{\operatorname{Re} p \rightarrow \infty} p^{2}\left[H_{i}(p)+Q\left(x_{i}, p\right)\right],
$$

for $i=1, \ldots, N=N_{1}+N_{2}$. They form a system for initial values $n_{k}(0)$ of the unknowns $n_{k}$, which has a unique solution provided

$$
\begin{aligned}
& \operatorname{rank}\left(\left(\frac{1}{\beta\left(x_{i}\right)} \nu_{k}\left(x_{i}\right) \varphi\left(x_{i}\right)\right)_{k=1, \ldots, N \text { i }} \lim _{\operatorname{Re} p \rightarrow \infty} p^{2}\left[H_{i}(p)+Q\left(x_{i}, p\right)\right]\right)_{i=1, \ldots, N} \\
& =\operatorname{rank}\left(\frac{1}{\beta\left(x_{i}\right)} \nu_{k}\left(x_{i}\right) \varphi\left(x_{i}\right)\right)_{\substack{k=1, \ldots, N_{1} \\
i=1, \ldots, N}}=N_{1} \text {. }
\end{aligned}
$$

Here the argument of the rank-function is a matrix, formed by placing two argument-matrices left to right. Thus, (4.3) is a necessary condition for the inverse problem to have a unique solution with the properties $1-3$.

We observe that the factors

$$
\int_{0}^{1} p G\left(x_{i}, y, p\right) \nu_{j}(y) p U(y, p) d y, \quad \sqrt{p} \int_{0}^{1} G_{y}\left(x_{i}, y, p\right) \mu_{k}(y) p U_{y}(y, p) d y
$$

of the terms $p N_{j}(p)$ and $\sqrt{p} M_{k}(p)$, respectively, in left-hand side of the system (4.1) are bounded on $\operatorname{Re} p>\sigma$ due to Lemma 1 below and the relations

$$
p U(y, p) \rightarrow \varphi(y), \quad p U_{y}(y, p) \rightarrow \varphi^{\prime}(y) \text { as } \operatorname{Re} p \rightarrow \infty .
$$

This suggests that the kernels $n_{k}$ and $m_{l}$ can be determined simultaneously with higher smoothness in $n_{k}$ than in $m_{l}$, because the exponent of $p$ is at $N_{j}(p)$ higher than at $M_{k}(9)$. Therefore we define the unknowns $Z=\left(Z_{1}, \ldots, Z_{N}\right)$, where

$$
Z_{k}(p)= \begin{cases}p N_{k}-n_{k}(0), & k=1, \ldots, N_{1}, \\ M_{k-N_{1}}(p), & k=N_{1}+1, \ldots, N,\end{cases}
$$

and derive a fixed point system for $Z$. To this end we rewrite (4.1) in the form 


$$
\begin{aligned}
\sum_{k=1}^{N_{1}} Z_{k}(p) \frac{1}{\beta\left(x_{i}\right)} \nu_{k}\left(x_{i}\right) \varphi\left(x_{i}\right)+\left.\sum_{k=N_{1}+1}^{N} Z_{k}(p) \frac{1}{\beta\left(x_{i}\right)}\left(\mu_{k-N_{1}}(x) \varphi^{\prime}(x)\right)^{\prime}\right|_{x=x_{i}} \\
\quad=\sum_{k=1}^{N_{1}} Z_{k}(p)\left[\int_{0}^{1} p G\left(x_{i}, y, p\right) \nu_{k}(y)[p U(y, p)-\varphi(y)] d y\right. \\
\left.\quad+\int_{0}^{1} p G\left(x_{i}, y, p\right) \nu_{k}(y) \varphi(y) d y+\frac{1}{\beta\left(x_{i}\right)} \nu_{k}\left(x_{i}\right) \varphi\left(x_{i}\right)\right] \\
\quad-\sum_{k=N_{1}+1}^{N} Z_{k}(p)\left[\int_{0}^{1} p G_{y}\left(x_{i}, y, p\right) \mu_{k-N_{1}}(y)\left[p U_{y}(y, p)-\varphi^{\prime}(y)\right] d y\right. \\
\quad-\int_{0}^{1} p G\left(x_{i}, y, p\right)\left(\mu_{k-N_{1}}(y) \varphi^{\prime}(y)\right)^{\prime} d y-\left.\frac{1}{\beta\left(x_{i}\right)}\left(\mu_{k-N_{1}}(x) \varphi^{\prime}(x)\right)^{\prime}\right|_{x=x_{i}} \\
\left.\quad+\mu_{k-N_{1}}(0) \varphi^{\prime}(0) p G\left(x_{i}, 0, p\right)-\mu_{k-N_{1}}(1) \varphi^{\prime}(1) p G\left(x_{i}, 1, p\right)\right] \\
\quad+\sum_{k=1}^{N_{1}} n_{k}(0) \int_{0}^{1} p G\left(x_{i}, y, p\right) \nu_{k}(y)[p U(y, p)-\varphi(y)] d y \\
\quad-p^{2}\left[H_{i}(p)+Q\left(x_{i}, p\right)\right]+\lim _{\operatorname{Re} q \rightarrow \infty} q^{2}\left[H_{i}(q)+Q\left(x_{i}, q\right)\right], i=1, \ldots, N . \quad(4.5)
\end{aligned}
$$

Indeed, opening the brackets in (4.5), integrating by parts the integral $\int_{0}^{1} p G\left(x_{i}, y, p\right)\left(\mu_{k-N_{1}}(y) \varphi^{\prime}(y)\right)^{\prime} d y$, simplifying, and observing (4.2), (4.4) it is not difficult to see that (4.5) is equivalent to (4.1).

In view of assertion (5.8) of Lemma 2 below and the relation $p U \rightarrow \varphi$ as $\operatorname{Re} p \rightarrow \infty$ the left-hand side of (4.5) is the principal part of this system. Therefore we introduce the matrix $\Gamma=\left(\gamma_{i k}\right)_{i, k=1, \ldots, N}$ related to this principal part, where

$$
\gamma_{i k}= \begin{cases}\frac{1}{\beta\left(x_{i}\right)} \nu_{k}\left(x_{i}\right) \varphi\left(x_{i}\right), & k=1, \ldots, N_{1}, \\ \left.\frac{1}{\beta\left(x_{i}\right)}\left(\mu_{k-N_{1}}(y) \varphi^{\prime}(y)\right)^{\prime}\right|_{y=x_{i}}, & k=N_{1}+1, \ldots, N\end{cases}
$$

and assume $\operatorname{det} \Gamma \neq 0$.

Further, we introduce vanishing with $\operatorname{Re} p \rightarrow \infty$ functions

$$
B^{0}[Z](x, p)=p U[Z](x, p)-\varphi(x), \quad B^{1}[Z](x, p)=p U_{x}[Z](x, p)-\varphi^{\prime}(x),
$$

where $U[Z](x, p)$ is the Laplace transform of the $Z$-dependent solution of the direct problem. Now system (4.5) can be written in the fixed-point form

$$
Z=\Gamma^{-1} \mathcal{F}(Z)
$$


where $\mathcal{F}(Z)=\left(\mathcal{F}_{1}(Z), \ldots, \mathcal{F}_{N}(Z)\right)$,

$$
\begin{aligned}
\mathcal{F}_{i}[Z](p) & =\sum_{k=1}^{N_{1}} Z_{k}(p)\left[\int_{0}^{1} p G\left(x_{i}, y, p\right) \nu_{k}(y) B^{0}[Z](y, p) d y\right. \\
& \left.+\int_{0}^{1} p G\left(x_{i}, y, p\right) \nu_{k}(y) \varphi(y) d y+\frac{1}{\beta\left(x_{i}\right)} \nu_{k}\left(x_{i}\right) \varphi\left(x_{i}\right)\right] \\
& +\sum_{k=N_{1}+1}^{N} Z_{k}(p)\left[-\int_{0}^{1} p G_{y}\left(x_{i}, y, p\right) \mu_{k-N_{1}}(y) B^{1}[Z](y, p) d y\right. \\
& +\int_{0}^{1} p G\left(x_{i}, y, p\right)\left(\mu_{k-N_{1}}(y) \varphi^{\prime}(y)\right)^{\prime} d y+\left.\frac{1}{\beta\left(x_{i}\right)}\left(\mu_{k-N_{1}}(x) \varphi^{\prime}(x)\right)^{\prime}\right|_{x=x_{i}} \\
& \left.+\mu_{k-N_{1}}(0) \varphi^{\prime}(0) p G\left(x_{i}, 0, p\right)-\mu_{k-N_{1}}(1) \varphi^{\prime}(1) p G\left(x_{i}, 1, p\right)\right] \\
& +\sum_{k=1}^{N_{1}} n_{k}(0) \int_{0}^{1} p G\left(x_{i}, y, p\right) \nu_{k}(y) B^{0}[Z](y, p) d y+\widehat{\Psi}_{i}(p), i=1, \ldots, N, \quad(4.9) \\
\widehat{\Psi}_{i}(p)= & \sum_{k=1}^{N_{1}} n_{k}(0)\left[\int_{0}^{1} p G\left(x_{i}, y, p\right) \nu_{k}(y) \varphi(y) d y+\frac{1}{\beta\left(x_{i}\right)} \nu_{k}\left(x_{i}\right) \varphi\left(x_{i}\right)\right] \\
& -p^{2}\left[H_{i}(p)+Q\left(x_{i}, p\right)\right]+\lim _{\operatorname{Re} q \rightarrow \infty} q^{2}\left[H_{i}(q)+Q\left(x_{i}, q\right)\right] .
\end{aligned}
$$

For future analysis we need a proper fixed-point system for the quantities $B^{0}[Z]$ and $B^{1}[Z]$, too. From (3.6) in view of the definitions of $Z$ and $B^{0}[Z]$ we have

$$
\begin{aligned}
B^{0}[Z](x, p) & =\sum_{k=1}^{N_{1}} Z_{k}(p) \int_{0}^{1} G(x, y, p) \nu_{k}(y)\left[B^{0}[Z](y, p)+\varphi(y)\right] d y \\
& -\sum_{k=N_{1}+1}^{N} Z_{k}(p) \int_{0}^{1} G_{y}(x, y, p) \mu_{k-N_{1}}(y)\left[B^{1}[Z](y, p)+\varphi^{\prime}(y)\right] d y \\
& +\sum_{k=1}^{N_{1}} n_{k}(0) \int_{0}^{1} G(x, y, p) \nu_{k}(y) B^{0}[Z](y, p) d y+\Phi^{0}(x, p)
\end{aligned}
$$

with

$$
\Phi^{0}(x, p)=\sum_{k=1}^{N_{1}} n_{k}(0) \int_{0}^{1} G(x, y, p) \nu_{k}(y) \varphi(y) d y-p Q(x, p)-\varphi(x) .
$$

From (3.9) we obtain 


$$
\begin{aligned}
B^{1}[Z](x, p) & =\sum_{k=1}^{N_{1}} Z_{k}(p) \int_{0}^{1} G_{x}(x, y, p) \nu_{k}(y)\left[B^{0}[Z](y, p)+\varphi(y)\right] d y \\
& +\sum_{k=N_{1}+1}^{N} Z_{k}(p)\left[\frac{\mu_{k-N_{1}}(x)}{\lambda(x)} B^{1}[Z](x, p)-\int_{0}^{1} G_{x y}(x, y, p) \mu_{k-N_{1}}(y)\right. \\
& \left.\times\left[B^{1}[Z](y, p)+\varphi^{\prime}(y)\right]\right]+\sum_{k=1}^{N_{1}} n_{k}(0) \int_{0}^{1} G_{x}(x, y, p) \nu_{k}(y) B^{0}[Z](y, p) d y \\
& +\sum_{k=N_{1}+1}^{N} Z_{k}(p) \frac{\mu_{k-N_{1}}(x) \varphi^{\prime}(x)}{\lambda(x)}+\Phi^{1}(x, p)
\end{aligned}
$$

with

$$
\Phi^{1}(x, p)=\sum_{k=1}^{N_{1}} n_{k}(0) \int_{0}^{1} G_{x}(x, y, p) \nu_{k}(y) \varphi(y) d y-p Q_{x}(x, p)-\varphi^{\prime}(x) .
$$

For the function $B^{0}[Z]$, which in contrast to $B^{1}[Z]$ doesn't contain a space derivative of $U$, we need a certain higher regularity in the time variable. To this end we will assume that the free term $\Phi^{0}$ can be decomposed as follows

$$
\Phi^{0}(x, p)=\widetilde{B}^{0}(x, p)+\widetilde{\Phi}^{0}(x, p),
$$

where $\left|\widetilde{B}^{0}(x, p)\right| \leq \frac{\text { Const }}{|p|}$ and $\left|\widetilde{\Phi}^{0}(x, p)\right| \leq \frac{\text { Const }}{|p|^{\alpha}}$ with some $\alpha>1$ for $\operatorname{Re} p>$ $\sigma_{0}, x \in[0,1]$ and split $B^{0}[Z]$ into the sum

$$
B^{0}[Z](x, p)=\widetilde{B}^{0}(x, p)+\widehat{B}^{0}[Z](x, p),
$$

where for $\widehat{B}^{0}$ we will require that $\left|\widehat{B}^{0}[Z](x, p)\right| \leq \frac{\text { Const }}{|p|^{\alpha}}$ for $\operatorname{Re} p>\sigma_{0}$, $x \in[0,1]$.

From (4.11) and (4.12) in view of (4.15), (4.16) and the definitions of $Z$ and $B^{1}[Z]$ we deduce the following fixed-point equation for the vector $B[Z]=$ $\left(\widehat{B}^{0}[Z], B^{1}[Z]\right)$ :

$$
B[Z]=A[Z] B[Z]+b[Z],
$$

where $A[Z]=\left(A^{0}[Z], A^{1}[Z]\right)$ is the $Z$-dependent linear operator of $B$ with the components

$$
\begin{aligned}
\left(A^{0}[Z] B\right)(x, p) & =\sum_{k=1}^{N_{1}}\left(Z_{k}(p)+n_{k}(0)\right) \int_{0}^{1} G(x, y, p) \nu_{k}(y) \widehat{B}^{0}(y, p) d y \\
& -\sum_{k=N_{1}+1}^{N} Z_{k}(p) \int_{0}^{1} G_{y}(x, y, p) \mu_{k-N_{1}}(y) B^{1}(y, p) d y,
\end{aligned}
$$




$$
\begin{aligned}
& \left(A^{1}[Z] B\right)(x, p)=\sum_{k=1}^{N_{1}}\left(Z_{k}(p)+n_{k}(0)\right) \int_{0}^{1} G_{x}(x, y, p) \nu_{k}(y) \widehat{B}^{0}(y, p) d y \\
& +\sum_{k=N_{1}+1}^{N} Z_{k}(p)\left[\frac{\mu_{k-N_{1}}(x)}{\lambda(x)} B^{1}(x, p)-\int_{0}^{1} G_{x y}(x, y, p) \mu_{k-N_{1}}(y) B^{1}(y, p) d y\right]
\end{aligned}
$$

and $b[Z]=\left(b^{0}[Z], b^{1}[Z]\right)$ is the $Z$-dependent $B$-free term with the components

$$
\begin{aligned}
& b^{0}[Z](x, p)=\sum_{k=1}^{N_{1}} Z_{k}(p) \int_{0}^{1} G(x, y, p) \nu_{k}(y)\left[\widetilde{B}^{0}(y, p)+\varphi(y)\right] d y \\
& -\sum_{k=N_{1}+1}^{N} Z_{k}(p) \int_{0}^{1} G_{y}(x, y, p) \mu_{k-N_{1}}(y) \varphi^{\prime}(y) d y+\widehat{\Phi}^{0}(x, p) \\
& b^{1}[Z](x, p)=\sum_{k=1}^{N_{1}} Z_{k}(p) \int_{0}^{1} G_{x}(x, y, p) \nu_{k}(y)\left[\widetilde{B}^{0}(y, p)+\varphi(y)\right] d y+\sum_{k=N_{1}+1}^{N} Z_{k}(p) \\
& \times\left[\frac{\mu_{k-N_{1}}(x) \varphi^{\prime}(x)}{\lambda(x)}-\int_{0}^{1} G_{x y}(x, y, p) \mu_{k-N_{1}}(y) \varphi^{\prime}(y) d y\right]+\widehat{\Phi}^{1}(x, p)
\end{aligned}
$$

and

$$
\begin{aligned}
\widehat{\Phi}^{0}(x, p) & =\sum_{k=1}^{N_{1}} n_{k}(0) \int_{0}^{1} G(x, y, p) \nu_{k}(y) \widetilde{B}^{0}(y, p) d y+\widetilde{\Phi}^{0}(x, p), \\
\widehat{\Phi}^{1}(x, p) & =\sum_{k=1}^{N_{1}} n_{k}(0) \int_{0}^{1} G_{x}(x, y, p) \nu_{k}(y)\left[\widetilde{B}^{0}(y, p)+\varphi(y)\right] d y \\
& -p Q_{x}(x, p)-\varphi^{\prime}(x) .
\end{aligned}
$$

\section{Functional Spaces and Estimation of Green Function}

To analyse the direct and inverse problems we define the spaces

$$
\mathcal{A}_{\gamma, \sigma}=\left\{V: V(p) \text { is holomorphic on } \operatorname{Re} p>\sigma,\|V\|_{\gamma, \sigma}<\infty\right\},
$$

where

$$
\|V\|_{\gamma, \sigma}=\sup _{\operatorname{Re} p>\sigma}|p|^{\gamma}|V(p)|
$$

and

$$
\left(\mathcal{A}_{\gamma, \sigma}\right)^{N}=\left\{V=\left(V_{1}, \ldots, V_{N}\right): V_{k}(p) \in \mathcal{A}_{\gamma, \sigma}, k=1, \ldots, N\right\}
$$


with the norm

$$
\|V\|_{\gamma, \sigma}=\sum_{k=1}^{N}\left\|V_{k}\right\|_{\gamma, \sigma}, \quad V \in\left(\mathcal{A}_{\gamma, \sigma}\right)^{N}
$$

We note that $\mathcal{A}_{\gamma, \sigma} \subset \mathcal{A}_{\gamma, \sigma^{\prime}},\left(\mathcal{A}_{\gamma, \sigma}\right)^{N} \subset\left(\mathcal{A}_{\gamma, \sigma^{\prime}}\right)^{N}$ and $\|\cdot\|_{\gamma, \sigma^{\prime}} \leq\|\cdot\|_{\gamma, \sigma}$ if $\sigma^{\prime}>\sigma$.

Let $\alpha$ be a real number such that

$$
1<\alpha<\frac{3}{2}
$$

Moreover, let $c=\left(c_{1}, \ldots, c_{N}\right)$ be a given vector. We will search the solution $Z=\left(Z_{1}, \ldots, Z_{N}\right)$ of (4.10) from the space

$$
\mathcal{M}_{c, \sigma}=\left\{Z: Z=\frac{c}{p}+V(p), V \in\left(\mathcal{A}_{\alpha, \sigma}\right)^{N}\right\} .
$$

Furthermore, we introduce the spaces of $x$ - and $p$-dependent functions

$$
\begin{array}{r}
\mathcal{B}_{\gamma, \sigma}=\left\{F(x, p): F(x, \cdot) \in \mathcal{A}_{\gamma, \sigma} \text { for } x \in[0,1],\right. \\
F(\cdot, p) \in C[0,1] \text { for } \operatorname{Re} p>\sigma\}
\end{array}
$$

with the norms

$$
\|F\|_{\gamma, \sigma}=\max _{0 \leq x \leq 1} \sup _{\operatorname{Re} p>\sigma}|p|^{\gamma}|F(x, p)| .
$$

We are going to solve the equation (4.17) for the pair $B=\left(\widehat{B}^{0}, B^{1}\right)$ in the space $\mathcal{B}_{\sigma}=\mathcal{B}_{\alpha, \sigma} \times \mathcal{B}_{\alpha-\frac{1}{2}, \sigma}$ with the norm

$$
\|B\|_{\sigma}=\left\|\widehat{B}^{0}\right\|_{\alpha, \sigma}+\left\|B^{1}\right\|_{\alpha-\frac{1}{2}, \sigma}
$$

For estimating the Green function we use the following lemmas.

Lemma 1. Let $\lambda, \beta \in C^{2}[0,1]$ and $\lambda, \beta>0$ in $[0,1]$. Then

$$
\begin{aligned}
& K_{1}=\sup _{\substack{0 \leq x \leq 1 \\
\operatorname{Re} p>0}}|p| \int_{0}^{1}|G(x, y, p)| d y<\infty, \\
& K_{2}=\sup _{\substack{0 \leq x \leq 1 \\
\operatorname{Re} p>0}} \sqrt{|p|} \int_{0}^{1}\left|G_{x}(x, y, p)\right| d y<\infty, \\
& K_{3}=\sup _{\substack{0 \leq x \leq 1 \\
\operatorname{Re} p>0}} \sqrt{|p|} \int_{0}^{1}\left|G_{y}(x, y, p)\right| d y<\infty, \\
& K_{4}=\sup _{\substack{0 \leq x \leq 1 \\
\operatorname{Re} p>0}} \int_{0}^{1}\left|G_{x y}(x, y, p)\right| d y<\infty .
\end{aligned}
$$


Moreover,

$$
\begin{aligned}
& \sqrt{|p|}|G(x, 0, p)| \leq \bar{C}_{1} e^{-\bar{C}_{2} \sqrt{|p|} x} \quad \forall x \in[0,1], \quad \operatorname{Re} p>0, \\
& \sqrt{|p|}|G(x, 1, p)| \leq \bar{C}_{3} e^{-\bar{C}_{4} \sqrt{|p|}(1-x)} \quad \forall x \in[0,1], \quad \operatorname{Re} p>0,
\end{aligned}
$$

where $\bar{C}_{1}, \bar{C}_{2}, \bar{C}_{2}, \bar{C}_{2}$ are certain positive constants.

Proof. The assertions (5.3)-(5.6) were proved in [4]. To prove (5.7), (5.8) we use the following representation for $G$ (see [4]):

$$
G(x, y ; p)=\frac{1}{C_{0}(p)} \frac{1}{a(x) a(y)}\left\{\begin{array}{l}
\operatorname{ch}(s z) \operatorname{ch}(s(1-w))+O_{1}, \text { for } x \leq y \\
\operatorname{ch}(s w) \operatorname{ch}(s(1-z))+O_{2}, \text { for } y \leq x .
\end{array}\right.
$$

Here $s=l \sqrt{p}, C_{0}(p)=-\frac{s}{l} \operatorname{sh}(s)+O_{3}, a(x)=\sqrt[4]{\beta(x) \lambda(x)}$,

$$
z=\frac{1}{l} \int_{0}^{x} \sqrt{\frac{\beta(\eta)}{\lambda(\eta)}} d \eta, w=\frac{1}{l} \int_{0}^{y} \sqrt{\frac{\beta(\eta)}{\lambda(\eta)}} d \eta, l=\int_{0}^{1} \sqrt{\frac{\beta(\eta)}{\lambda(\eta)}} d \eta
$$

and

$$
O_{1}=\mathcal{O}\left(\frac{e^{s(1-w+z)}}{s}\right), O_{2}=\mathcal{O}\left(\frac{e^{s(w+1-z)}}{s}\right), O_{3}=\mathcal{O}\left(e^{s}\right)
$$

for $\operatorname{Re} p \rightarrow+\infty$ holding uniformly in $x$ and $y$ from $[0,1]$ and $\operatorname{Im} p \in \mathbb{R}$. According to this representation we have

$$
G(x, 0, p)=-\frac{l}{s a(x) a(0)} \frac{\operatorname{ch}(s(1-z))+\mathcal{O}\left(\frac{e^{s(1-z)}}{s}\right)}{\operatorname{sh}(s)+\mathcal{O}\left(\frac{e^{s}}{s}\right)} \text { for } \quad \operatorname{Re} p \rightarrow \infty,
$$

where the $\mathcal{O}$-terms are uniform in $z$ and $\operatorname{Im} p$. Since $|s|=l \sqrt{|p|} \rightarrow \infty$ as $\operatorname{Re} p \rightarrow \infty$ from this relation and the local boundedness of $G$ we obtain the estimate

$$
|G(x, 0, p)| \leq \bar{C}_{5}|s|^{-1}\left|e^{-s z}\right|, \text { for } \operatorname{Re} p>0, x \in[0,1]
$$

with some nonnegative constant $\bar{C}_{5}$. Further, using the definition of $z$ and the relation $\operatorname{Re} s=l \operatorname{Re} \sqrt{p}>\frac{l}{\sqrt{2}} \sqrt{|p|}=\frac{1}{\sqrt{2}}|s|$, which holds for $\operatorname{Re} p>0$, we deduce

$$
|G(x, 0, p)| \leq \bar{C}_{5}\left|\frac{e^{-s z}}{|s|}\right|=\bar{C}_{5} \frac{e^{-\operatorname{Re} s z}}{|s|}<\bar{C}_{5} \frac{e^{-\frac{|s|}{\sqrt{2}} z}}{|s|} \leq \bar{C}_{4} \frac{e^{-\bar{C}_{2} \sqrt{|p|} x}}{l \sqrt{|p|}}
$$

with some constant $\bar{C}_{2}>0$. This implies (5.7). The estimate (5.8) can be proved in a similar manner. 
Lemma 2. Let $\lambda, \beta \in C^{2}[0,1], \lambda, \beta>0$ in $[0,1]$ and $v \in C^{1}[0,1]$. Then

$$
\sup _{\operatorname{Re} p>0}\left|\sqrt{|p|}\left(\int_{0}^{1} p G(x, y, p) v(y) d y+\frac{v(x)}{\beta(x)}\right)\right| \leq K_{5}\|v\|_{C^{1}[0,1]}
$$

for all $x \in(0,1)$, where $K_{5}$ is independent of $x$ in every closed subinterval of $(0,1)$.

Lemma 2 was proved in [4].

\section{Analysis of Direct Problem}

Let us assume

$\lambda, \beta \in C^{2}[0,1], \quad \lambda, \beta>0 ;$

$\Phi^{0}$ given by (4.12) admits the decomposition(4.15), where

$\widetilde{B}^{0} \in \mathcal{B}_{1, \sigma_{0}}$ and $\widetilde{\Phi}^{0} \in \mathcal{B}_{\alpha, \sigma_{0}}$ with some $\sigma_{0} \geq 1$ and $\alpha$ satisfying (5.1);

$\Phi^{1}$ given by (4.14) belongs to $\mathcal{B}_{\alpha-\frac{1}{2}, \sigma_{0}}, \varphi \in C^{1}[0,1]$;

$\nu_{k} \in C[0,1], k=1, \ldots, N_{1}, \mu_{l} \in C[0,1], l=1, \ldots, N_{2}$.

Lemma 3. Let the assumptions (6.1) hold. If $Z=\frac{c}{p}+V \in \mathcal{M}_{c, \sigma}$ then the vector function $b[Z]=\left(b^{0}[Z], b^{1}[Z]\right)$, given by $(4.20)$, (4.21), belongs to $\mathcal{B}_{\sigma_{0}}$ and satisfies the estimate

$$
\|b[Z]\|_{\sigma} \leq C_{1}\left[1+\frac{1}{\sigma^{\frac{3}{2}-\alpha}}\left(|c|+\frac{\|V\|_{\alpha, \sigma}}{\sigma^{\alpha-1}}\right)\right]
$$

with any $\sigma \geq \sigma_{0}$, where $C_{1}$ is a constant and $|c|=\sum_{k=1}^{N}\left|c_{k}\right|$. Moreover, for every $\sigma \geq \sigma_{0}$ and $Z^{1}=\frac{c}{p}+V^{1}, Z^{2}=\frac{c}{p}+V^{2} \in \mathcal{M}_{c, \sigma}$ the difference $b\left[Z^{1}\right]-b\left[Z^{2}\right]$ fulfills the estimate

$$
\left\|b\left[Z^{1}\right]-b\left[Z^{2}\right]\right\|_{\sigma} \leq C_{2} \frac{1}{\sqrt{\sigma}}\left\|V^{1}-V^{2}\right\|_{\alpha, \sigma}
$$

with a constant $C_{2}$.

Lemma 4. Let the assumptions (6.1) hold. If $Z=\frac{c}{p}+V \in \mathcal{M}_{c, \sigma}$ then the linear operator $A[Z]=\left(A^{0}[Z], A^{1}[Z]\right)$, defined by (4.18), (4.19), is bounded in $\mathcal{B}_{\sigma}$ and satisfies the estimate

$$
\|A[Z]\|_{\mathscr{L}\left(\mathcal{B}_{\sigma}\right)} \leq C_{3}\left[\frac{1+|c|}{\sigma}+\frac{\|V\|_{\alpha, \sigma}}{\sigma^{\alpha}}\right]
$$


for any $\sigma \geq \sigma_{0}$ with a constant $C_{3}$. Moreover, taking $Z^{1}=\frac{c}{p}+V^{1}, Z^{2}=$ $\frac{c}{p}+V^{2} \in \mathcal{M}_{c, \sigma}$, the estimate for difference

$$
\left\|\left(A\left[Z^{1}\right]-A\left[Z^{2}\right]\right)\right\|_{\mathscr{L}\left(\mathcal{B}_{\sigma}\right)} \leq \frac{C_{4}}{\sigma^{\alpha}}\left\|V^{1}-V^{2}\right\|_{\alpha, \sigma}
$$

holds for any $\sigma \geq \sigma_{0}$ with a constant $C_{4}$.

Proofs of Lemmas 3 and 4 are shifted to the appendix of the paper.

Due to Lemmas 3, 4 and the contraction principle equation (4.17) has a unique solution $B=B[Z] \in \mathcal{B}_{\sigma}$ provided $Z=\frac{c}{p}+V \in \mathcal{M}_{c, \sigma}$ and $\sigma \geq \sigma_{0}$ satisfy the relation

$$
\eta(Z, \sigma):=\frac{1+|c|}{\sigma}+\frac{\|V\|_{\alpha, \sigma}}{\sigma^{\alpha}} \leq \frac{1}{2 C_{3}} .
$$

Furthermore, from (4.17) we have

$$
\|B[Z]\|_{\sigma} \leq\left(1-\|A[Z]\|_{\mathscr{L}\left(\mathcal{B}_{\sigma}\right)}\right)^{-1}\|b[Z]\|_{\sigma} .
$$

This in view of $(6.2),(6.4)$ and (6.6) yields the estimate

$$
\|B[Z]\|_{\sigma} \leq 2 C_{1}\left[1+\frac{1}{\sigma^{\frac{3}{2}-\alpha}}\left(|c|+\frac{\|V\|_{\alpha, \sigma}}{\sigma^{\alpha-1}}\right)\right]
$$

for the solution of (4.17).

Next let us find an estimate for $B\left[Z^{1}\right]-B\left[Z^{2}\right]$. Let $\sigma \geq \sigma_{0}$ and $Z^{1}=\frac{c}{p}+V^{1}, Z^{2}=\frac{c}{p}+V^{2}$ be such that (6.6) is valid for $V$ replaced by $V^{1}$ and $V^{2}$, i.e. $\eta\left(Z^{j}, \sigma\right) \leq \frac{1}{2 C_{3}}, j=1,2$. Subtracting equation (4.17) for $Z=Z^{2}$ from the corresponding equation for $Z=Z^{1}$ we have

$$
\begin{aligned}
B\left[Z^{1}\right]-B\left[Z^{2}\right]=A\left[Z^{2}\right]\left(B\left[Z^{1}\right]-B\left[Z^{2}\right]\right) & +\left(A\left[Z^{1}\right]-A\left[Z^{2}\right]\right) B\left[Z^{1}\right] \\
& +b\left[Z^{1}\right]-b\left[Z^{2}\right] .
\end{aligned}
$$

This implies

$$
\begin{aligned}
\| B\left[Z^{1}\right] & -B\left[Z^{2}\right] \|_{\sigma} \leq\left(1-\left\|A\left[Z^{2}\right]\right\|_{\mathscr{L}\left(\mathcal{B}_{\sigma}\right)}\right)^{-1} \\
& \times\left[\left\|A\left[Z^{1}\right]-A\left[Z^{2}\right]\right\|_{\mathscr{L}\left(\mathcal{B}_{\sigma}\right)}\left\|B\left[Z^{1}\right]\right\|_{\sigma}+\left\|b\left[Z^{1}\right]-b\left[Z^{2}\right]\right\|_{\sigma}\right] .
\end{aligned}
$$

Using in this relation the estimates (6.3)-(6.7) we obtain

$$
\begin{aligned}
\left\|B\left[Z^{1}\right]-B\left[Z^{2}\right]\right\|_{\sigma} \leq 2\left\{\frac{2 C_{1} C_{4}}{\sigma^{\alpha}}[1\right. & \left.\left.+\frac{1}{\sigma^{\frac{3}{2-\alpha}}}\left(|c|+\frac{\left\|V^{1}\right\|_{\alpha, \sigma}}{\sigma^{\alpha-1}}\right)\right]+\frac{C_{2}}{\sqrt{\sigma}}\right\} \\
& \times\left\|V^{1}-V^{2}\right\|_{\alpha, \sigma} .
\end{aligned}
$$

Summing up, we have proved the following theorem. 
Theorem 1. Let the assumptions (6.1) hold. Then for any $\sigma \geq \sigma_{0}$ and $Z=\frac{c}{p}+V \in \mathcal{M}_{c, \sigma}$, satisfying the inequality (6.6), equation (4.17) has a unique solution $B[Z]=\left(\widehat{B}^{0}[Z], B^{1}[Z]\right)$ in $\mathcal{B}_{\sigma}$. This solution satisfies estimate (6.7). Moreover, for every $\sigma \geq \sigma_{0}$ and $Z^{1}=\frac{c}{p}+V^{1}, Z^{2}=\frac{c}{p}+V^{2} \in \mathcal{M}_{c, \sigma}$ such that $\eta\left(Z^{j}, \sigma\right) \leq \frac{1}{2 C_{3}}, j=1,2$, the difference $B\left[Z^{1}\right]-B\left[Z^{2}\right]$ fulfills estimate (6.8).

\section{Existence and Uniqueness for Inverse Problem}

In this section we study the inverse problem in the fixed-point form (4.10) in the Laplace domain and thereupon infer a result for the inverse problem (2.1) $-(2.5)$ in the time domain.

Due to the decomposition (4.16) the full $Z$-free term of the operator $\mathcal{F}=$ $\left(\mathcal{F}_{1}, \ldots, \mathcal{F}_{N}\right)$ given by $(4.9)$ is $\Psi=\left(\Psi_{1}, \ldots, \Psi_{N}\right)$, where

$$
\Psi_{i}(p)=\widehat{\Psi}_{i}(p)+\sum_{k=1}^{N_{1}} n_{k}(0) \int_{0}^{1} p G\left(x_{i}, y, p\right) \nu_{k}(y) \widetilde{B}^{0}(y, p) d y
$$

and $\widehat{\Psi}_{i}$ is defined in (4.10).

Theorem 2. Assume that (6.1) holds and

$$
\nu_{k} \in C^{1}[0,1], k=1, \ldots, N_{1}, \mu_{l} \in C^{2}[0,1], l=1, \ldots, N_{2} ; \varphi \in C^{3}[0,1] .
$$

Moreover, let $\operatorname{det} \Gamma \neq 0$ for $\Gamma$, given by (4.6), and

$$
\Psi=\frac{d}{p}+Y \in \mathcal{M}_{d, \sigma_{0}}
$$

with some $d \in \mathbb{R}^{N}$. Then there exists $\sigma_{1} \geq \sigma_{0}$ such that equation (4.8) has a unique solution $Z=\frac{c}{p}+V \in \mathcal{M}_{c, \sigma_{1}}$, here $c=\Gamma^{-1} d$.

Proof. Setting $c=\Gamma^{-1} d$ and observing (7.1), (7.3), problem (4.8) for $Z=$ $\frac{c}{p}+V$ in $\mathcal{M}_{c, \sigma}$ is equivalent to the following equation for $V$ in $\left(\mathcal{A}_{\alpha, \sigma}\right)^{N}$ :

$$
V=F(V)
$$

where $F=\Gamma^{-1} F_{1}$,

$$
F_{1}(V)=L_{0}\left(\frac{c}{p}+V, B[Z]\right)+L_{1}\left(\frac{c}{p}+V\right)+L_{2}(B[Z])+Y,
$$

$L_{0}$ is the following bilinear operator of $Z \in \mathcal{M}_{c, \sigma}, B=\left(\widehat{B}^{0}, B^{1}\right) \in \mathcal{B}_{\sigma}$ : 


$$
\begin{aligned}
& \left(L_{0}(Z, B)\right)_{i}(p)=\sum_{k=1}^{N_{1}} Z_{k}(p) \int_{0}^{1} p G\left(x_{i}, y, p\right) \nu_{k}(y) \widehat{B}^{0}(y, p) d y \\
& -\sum_{k=N_{1}+1}^{N} Z_{k}(p) \int_{0}^{1} p G_{y}\left(x_{i}, y, p\right) \mu_{k-N_{1}}(y) B^{1}(y, p) d y, \quad i=1, \ldots, N
\end{aligned}
$$

and $L_{1}$ and $L_{2}$ are the linear operators of $Z \in \mathcal{M}_{c, \sigma}$ and $B=\left(\widehat{B}^{0}, B^{1}\right) \in \mathcal{B}_{\sigma}$, given by the formulae

$$
\begin{aligned}
& \left(L_{1}(Z)\right)_{i}(p)=\sum_{k=1}^{N_{1}} Z_{k}(p)\left\{\int_{0}^{1} p G\left(x_{i}, y, p\right) \nu_{k}(y) \widetilde{B}^{0}(y, p) d y\right. \\
& \left.\quad+\int_{0}^{1} p G\left(x_{i}, y, p\right) \nu_{k}(y) \varphi(y) d y+\frac{1}{\beta\left(x_{i}\right)} \nu_{k}\left(x_{i}\right) \varphi\left(x_{i}\right)\right\}+\sum_{k=N_{1}+1}^{N} Z_{k}(p) \\
& \quad \times\left\{\int_{0}^{1} p G\left(x_{i}, y, p\right)\left(\mu_{k-N_{1}}(y) \varphi^{\prime}(y)\right)^{\prime} d y+\left.\frac{1}{\beta\left(x_{i}\right)}\left(\mu_{k-N_{1}}(x) \varphi^{\prime}(x)\right)^{\prime}\right|_{x=x_{i}}\right. \\
& \left.+\mu_{k-N_{1}}(0) \varphi^{\prime}(0) p G\left(x_{i}, 0, p\right)-\mu_{k-N_{1}}(1) \varphi^{\prime}(1) p G\left(x_{i}, 1, p\right)\right\}, i=1, \ldots, N
\end{aligned}
$$

and

$$
\left(L_{2}(B)\right)_{i}(p)=\sum_{k=1}^{N_{1}} n_{k}(0) \int_{0}^{1} p G\left(x_{i}, y, p\right) \nu_{k}(y) \widehat{B}^{0}(y, p) d y, i=1, \ldots, N,
$$

respectively. We will prove the assertion of theorem using the fixed-point argument in the following balls:

$$
D_{\alpha, \sigma}(\rho)=\left\{V \in\left(\mathcal{A}_{\alpha, \sigma}\right)^{N}:\|V\|_{\alpha, \sigma} \leq \rho\right\} .
$$

To this end we first deduce some estimates for $L_{0}, L_{1}$ and $L_{2}$. Multiplying by $|p|^{\alpha}$ in (7.6) and estimating we have

$$
\begin{aligned}
& |p|^{\alpha}\left|\left(L_{0}\left(\frac{c}{p}+V, B\right)\right)_{i}(p)\right| \leq \sum_{k=1}^{N_{1}}\left(\frac{\left|c_{k}\right|}{|p|}+\frac{|p|^{\alpha}\left|V_{k}(p)\right|}{|p|^{\alpha}}\right)|p| \\
& \quad \int_{0}^{1}\left|G\left(x_{i}, y, p\right)\right| d y \times\left\|\nu_{k}(y)\right\|_{C[0,1]}|p|^{\alpha} \max _{0 \leq y \leq 1}\left|\widehat{B}^{0}(y, p)\right| \\
& \quad+\sum_{k=N_{1}+1}^{N}\left(\left|c_{k}\right|+\frac{|p|^{\alpha}\left|V_{k}(p)\right|}{|p|^{\alpha-1}}\right) \sqrt{|p|} \int_{0}^{1}\left|G_{y}\left(x_{i}, y, p\right)\right| d y \\
& \quad \times\left\|\mu_{k-N_{1}}\right\|_{C[0,1]}|p|^{\alpha-\frac{1}{2}} \max _{0 \leq y \leq 1}\left|B^{1}(y, p)\right|, i=1, \ldots, N .
\end{aligned}
$$


Using the assumptions (6.1), (7.2), the assertions (5.3), (5.5) of Lemma 1 and the definition of the norm $\|\cdot\|_{\gamma, \sigma}$ we obtain

$$
\begin{array}{r}
|p|^{\alpha}\left|\left(L_{0}\left(\frac{c}{p}+V, B\right)\right)_{i}(p)\right| \leq \sum_{k=1}^{N_{1}}\left(\frac{\left|c_{k}\right|}{|p|}+\frac{\left\|V_{k}\right\|_{\alpha, \sigma}}{|p|^{\alpha}}\right) K_{1}\left\|\nu_{k}(y)\right\|_{C[0,1]}\left\|\widehat{B}^{0}\right\|_{\alpha, \sigma} \\
\quad+\sum_{k=N_{1}+1}^{N}\left(\left|c_{k}\right|+\frac{\left\|V_{k}\right\|_{\alpha, \sigma}}{|p|^{\alpha-1}}\right) K_{3}\left\|\mu_{k-N_{1}}\right\|_{C[0,1]}\left\|B^{1}\right\|_{\alpha-\frac{1}{2}, \sigma}, i=1, \ldots, N
\end{array}
$$

for $\operatorname{Re} p>\sigma, \sigma \geq \sigma_{0}, x \in[0,1]$. Taking in this relation the supremum over $\operatorname{Re} p>\sigma, x \in[0,1]$, observing the relation $|p|^{\gamma}>\sigma^{\gamma}$ for $\operatorname{Re} p>\sigma$, which holds in the cases $\gamma=1, \alpha, \alpha-1$ due to the assumed inequality (5.1) and the inequality $1 / \sigma \leq 1$, which holds due to $\sigma \geq \sigma_{0} \geq 1$, we get

$$
\left\|L_{0}\left(\frac{c}{p}+V, B\right)\right\|_{\alpha, \sigma} \leq C_{6}\left(|c|+\frac{\|V\|_{\alpha, \sigma}}{\sigma^{\alpha-1}}\right)\|B\|_{\sigma}, \sigma \geq \sigma_{0},
$$

where $C_{6}$ is a constant. Similarly, from (7.7) by means of the assertions (5.3), (5.7), (5.8) and (5.9) of lemmas 1 and 2 we deduce

$$
\begin{aligned}
& |p|^{\alpha}\left|\left(L_{1}\left(\frac{c}{p}+V\right)\right)_{i}(p)\right| \leq \sum_{k=1}^{N_{1}}\left(\frac{\left|c_{k}\right|}{|p|^{\frac{3}{2}-\alpha}}+\frac{\left\|V_{k}\right\|_{\alpha, \sigma}}{\sqrt{|p|}}\right)\left(\frac{K_{1}}{\sqrt{|p|}}\left\|\nu_{k}\right\|_{C[0,1]}\left\|\widetilde{B}^{0}\right\|_{1, \sigma_{0}}\right. \\
& \left.\quad+K_{5}\left\|\nu_{k} \varphi\right\|_{C^{1}[0,1]}\right)+\sum_{k=N_{1}+1}^{N}\left(\frac{\left|c_{k}\right|}{|p|^{\frac{3}{2}-\alpha}}+\frac{\left\|V_{k}\right\|_{\alpha, \sigma}}{\sqrt{|p|}}\right) \\
& \quad \times\left(K_{5}\left\|\left(\mu_{k-N_{1}} \varphi^{\prime}\right)^{\prime}\right\|_{C^{1}[0,1]}+\bar{C}_{1}\left|\mu_{k-N_{1}}(0) \varphi^{\prime}(0)\right||p|^{\frac{3}{2}} e^{-\bar{C}_{2} \sqrt{|p|} x_{i}}\right. \\
& \left.\quad+\bar{C}_{3}\left|\mu_{k-N_{1}}(1) \varphi^{\prime}(1)\right||p|^{\frac{3}{2}} e^{-\bar{C}_{4} \sqrt{|p|}\left(1-x_{i}\right)}\right), \quad i=1, \ldots N,
\end{aligned}
$$

for $\operatorname{Re} p>\sigma, \sigma \geq \sigma_{0}, x \in[0,1]$. Let us define $\kappa=\min _{i=1, \ldots, N}\left\{\bar{C}_{2} x_{i} ; \bar{C}_{4}\left(1-x_{i}\right)\right\}$. Since $x_{i} \in(0,1), i=1, \ldots, N$, we have $\kappa>0$. Thus, there exists $\bar{\sigma}_{0} \geq \sigma_{0}$ such that the function $\chi(\sigma)=\sigma^{\frac{3}{2}} e^{-\kappa \sqrt{\sigma}}$ is decreasing for $\sigma \geq \bar{\sigma}_{0}$. Taking in (7.10) the supremum over $\operatorname{Re} p>\sigma, x \in[0,1]$, where $\sigma \geq \bar{\sigma}_{0}$, observing the relation $|p|^{\gamma}>\sigma^{\gamma}$ for $\operatorname{Re} p>\sigma$, which holds in the cases $\gamma=3 / 2-\alpha, \frac{1}{2}$ due to (5.1), and the monotonicity of $\chi(\sigma)$ we obtain

$$
\left\|L_{1}\left(\frac{c}{p}+V\right)\right\|_{\alpha, \sigma} \leq C_{7}\left(\frac{|c|}{\sigma^{\frac{3}{2}-\alpha}}+\frac{\|V\|_{\alpha, \sigma}}{\sqrt{\sigma}}\right), \sigma \geq \bar{\sigma}_{0},
$$

where $C_{7}$ is a constant. Finally, from (7.8) in view of (5.3) we deduce

$$
\left\|L_{2}(B)\right\|_{\alpha, \sigma} \leq C_{8}\|B\|_{\sigma}, \sigma \geq \sigma_{0},
$$

with a constant $C_{8}$.

Let us return to the equation (7.4) with the operator $F=\Gamma^{-1} F_{1}$, where $F_{1}$ is given by (7.5). By means of (7.9), (7.11), (7.12) we obtain 


$$
\left\|F_{1}(V)\right\|_{\alpha, \sigma} \leq C_{9}\left(1+\frac{\|V\|_{\alpha, \sigma}}{\sigma^{\alpha-1}}\right)\left(\|B[Z]\|_{\sigma}+\frac{1}{\sigma^{\frac{3}{2}-\alpha}}\right)+\|Y\|_{\sigma_{0}}, \sigma \geq \bar{\sigma}_{0},
$$

with a constant $C_{9}$. Further, let us suppose that $V \in D_{\alpha, \sigma}(\rho)$, where $\sigma \geq \bar{\sigma}_{0}$ and $\sigma, \rho$ satisfy the relation

$$
\eta_{0}(\rho, \sigma):=\frac{1+|c|}{\sigma}+\frac{\rho}{\sigma^{\alpha}} \leq \frac{1}{2 C_{3}} .
$$

Then (6.6) holds, hence we can apply estimate (6.7) of Theorem 1 for $\|B[Z]\|_{\sigma}$. Plugging (6.7) into (7.13) and estimating $\|V\|_{\alpha, \sigma}$ by $\rho$ and observing that $1 / \sigma^{\frac{3}{2}-\alpha} \leq$ const for $\sigma \geq \bar{\sigma}_{0}$ we have

$$
\left\|F_{1}(V)\right\|_{\alpha, \sigma} \leq C_{10}\left(1+\frac{\rho}{\sigma^{\alpha-1}}\right)^{2}+\|Y\|_{\sigma_{0}}
$$

with a constant $C_{10}$. From (7.10) due to the equality $F=\Gamma^{-1} F_{1}$ and the relation $\alpha>1$ we see that for every $\rho>\rho_{0}:=\left|\Gamma^{-1}\right|\left(C_{10}+\|Y\|_{\alpha, \sigma_{0}}\right)$ there exists $\sigma_{2}=\sigma_{2}(\rho) \geq \bar{\sigma}_{0}$ such that the inequalities $\eta_{0}(\rho, \sigma) \leq \frac{1}{2 C_{3}}$ and $\|F V\|_{\alpha, \sigma} \leq \rho$ hold for any $\sigma \geq \sigma_{2}(\rho)$. Consequently,

$$
F: D_{\alpha, \sigma}(\rho) \rightarrow D_{\alpha, \sigma}(\rho) \quad \text { for } \quad \rho>\rho_{0} \quad \text { and } \quad \sigma \geq \sigma_{2}(\rho) .
$$

Next, we prove that $F$ is a contraction. From (7.5) we have

$$
\begin{aligned}
F_{1}(V)-F_{1}(\tilde{V}) & =L_{0}(V-\widetilde{V}, B[Z])+L_{0}\left(\frac{c}{p}+\widetilde{V}, B[Z]-B[\widetilde{Z}]\right) \\
& +L_{1}(V-\widetilde{V})+L_{2}(B[Z]-B[\widetilde{Z}])
\end{aligned}
$$

where $Z=\frac{c}{p}+V, \widetilde{Z}=\frac{c}{p}+\widetilde{V}$. Using here (7.9), (7.11), (7.12) and observing the inequality $1 / \sqrt{\sigma} \leq 1 / \sigma^{\alpha-1}$ that holds due to $\sigma \geq \bar{\sigma}_{0} \geq 1$ and (5.1), we get

$$
\begin{aligned}
\left\|F_{1}(V)-F_{1}(\widetilde{V})\right\|_{\alpha, \sigma} & \leq C_{11}\left\{\frac{1}{\sigma^{\alpha-1}}\left(1+\|B[Z]\|_{\sigma}\right)\|V-\widetilde{V}\|_{\alpha, \sigma}\right. \\
& \left.+\left(1+\frac{\|\widetilde{V}\|_{\alpha, \sigma}}{\sigma^{\alpha-1}}\right)\|B[Z]-B[\widetilde{Z}]\|_{\sigma}\right\}, \sigma \geq \bar{\sigma}_{0},
\end{aligned}
$$

with a constant $C_{11}$. Let us suppose that $V, \widetilde{V} \in D_{\alpha, \sigma}(\rho)$ where $\sigma \geq \bar{\sigma}_{0}$ and $\rho, \sigma$ satisfy (7.14). Then we can make use of the estimates (6.7) and (6.8) of Theorem 1 and we have

$$
\begin{aligned}
& \left\|F_{1}(V)-F_{1}(\widetilde{V})\right\|_{\alpha, \sigma} \leq C_{12}\left\{\frac{1}{\sigma^{\alpha-1}}\left(1+\frac{1}{\sigma^{\frac{3}{2}-\alpha}}\left(1+\frac{\rho}{\sigma^{\alpha-1}}\right)\right)\right. \\
& \quad+\left(1+\frac{\rho}{\sigma^{\alpha-1}}\right)\left[\frac{1}{\sigma^{\alpha}}\left(1+\frac{1}{\sigma^{\frac{3}{2}-\alpha}}\left(1+\frac{\rho}{\sigma^{\alpha-1}}\right)\right)+\frac{1}{\sqrt{\sigma}}\right\}\|V-\widetilde{V}\|_{\alpha, \sigma} .
\end{aligned}
$$

In view of the relations $1<\alpha<\frac{3}{2}$ the coefficient of $\|V-\widetilde{V}\|_{\alpha, \sigma}$ on the right-hand side of this estimate approaches zero as $\sigma \rightarrow \infty$ for a fixed $\rho>0$. Hence, for every $\rho>0$ there exists $\sigma_{3}=\sigma_{3}(\rho) \geq \bar{\sigma}_{0}$, such that the inequality 
$\eta_{0}(\rho, \sigma) \leq 1 / 2 C_{3}$ holds and $F=\Gamma^{-1} F_{1}$ is a contraction in the ball $D_{\alpha, \sigma}(\rho)$ for $\rho>0$ and $\sigma \geq \sigma_{3}(\rho)$. This together with (7.16) shows that equation (7.4) has a unique solution $V$ in every ball $D_{\alpha, \sigma}(\rho)$, where $\rho>\rho_{0}$ and $\sigma \geq$ $\sigma_{4}(\rho)=\max \left\{\sigma_{2}(\rho) ; \sigma_{3}(\rho)\right\}$. This proves the existence assertion of theorem with $\sigma_{1}=\sigma_{4}\left(2 \rho_{0}\right)$.

It remains to prove that the solution of (7.4) is unique in the whole space $\left(\mathcal{A}_{\alpha, \sigma_{1}}\right)^{N}$. Suppose that (7.4) has two solutions $V^{1}$ and $V^{2}$ in $\left(\mathcal{A}_{\alpha, \sigma_{1}}\right)^{N}$. Let us define $\bar{\rho}:=\max \left(2 \rho_{0} ;\left\|V^{1}\right\|_{\alpha, \sigma_{1}} ;\left\|V^{2}\right\|_{\alpha, \sigma_{1}}\right)$ and $\bar{\sigma}:=\max \left(\sigma_{1} ; \sigma_{4}(\bar{\rho})\right)$. Then we have $\left\|V^{j}\right\|_{\alpha, \sigma_{1}} \leq \bar{\rho}, j=1,2$. Since the norm $\|\cdot\|_{\alpha, \sigma}$ is non-increasing with respect to $\sigma$ and $\bar{\sigma} \geq \sigma_{1}$, from this relation we derive

$$
\left\|V^{j}\right\|_{\alpha, \bar{\sigma}} \leq \bar{\rho} \quad \Longrightarrow \quad V^{j} \in D_{\alpha, \bar{\sigma}}(\bar{\rho}), \quad j=1,2 .
$$

But due to $\bar{\rho}>\rho_{0}$ and $\bar{\sigma} \geq \sigma_{4}(\bar{\rho})$, the uniqueness in the ball $D_{\alpha, \bar{\sigma}}(\bar{\rho})$ has already been shown. Thus, $V^{1}=V^{2}$. Theorem 2 is proved.

Finally, applying the well-known results about the invertability of the Laplace transform [3] we deduce the following corollary from Theorem 2.

Corollary 1. Let conditions (4.3) hold yielding the unique initial values $n_{j}(0)$ for the unknowns $n_{j}, k=1, \ldots, N_{1}$ from system (4.2). Moreover, let the assumptions of 2 be satisfied for the functions $\lambda_{k}, \mu_{l}, \varphi$ and the quantities $\Phi^{0}, \Phi^{1}, \Psi$ given by formulas (4.12), (4.14), (7.1) with (4.10), (4.14) in terms of the Laplace transforms $R, F_{1}, F_{2}, H_{i}$ of the data of inverse problem (2.1)(2.5). Then inverse problem (2.1)-(2.5) has the unique solution $(n, m)$ with coefficients $n_{j}$ and $m_{k}$ of the form

$$
\begin{aligned}
& n_{j}(t)=n_{j}(0)+c_{j} t+\frac{1}{2 \pi i} \int_{0}^{t} \int_{\xi-i \infty}^{\xi+i \infty} e^{\tau p} Z_{j}(p) d p d \tau, \quad k=1, \ldots N_{1}, \\
& m_{k}(t)=c_{k+N_{1}}+\frac{1}{2 \pi i} \int_{\xi-i \infty}^{\xi+i \infty} e^{t p} Z_{k+N_{1}}(p) d p, \quad k=1, \ldots N_{2},
\end{aligned}
$$

where $c=\left(c_{1}, \ldots, c_{N}\right) \in \mathbb{R}^{N}, Z=\left(Z_{1}, \ldots, Z_{N}\right) \in\left(\mathcal{A}_{\alpha, \sigma_{1}}\right)^{N}, N=N_{1}+N_{2}$. The functions $n_{j}$ are continuously differentiable and $m_{k}$ are continuous for $t \geq 0$. Moreover, $n_{j}^{\prime}(0)=c_{j}, j=1, \ldots, N_{1}$ and $m_{k}(0)=c_{k+N_{1}}, k=1, \ldots, N_{2}$.

\section{Appendix}

Proof of the Lemma 3 Let us start with the estimation of $b^{0}[Z]$. Substituting $\frac{c}{p}+V$ for $Z$ in (4.20), multiplying by $|p|^{\alpha}$ and estimating we have

$$
|p|^{\alpha}\left|b^{0}[Z](x, p)\right| \leq \sum_{k=1}^{N_{1}}\left(\left|c_{k}\right|+\frac{|p|^{\alpha}\left|V_{k}(p)\right|}{|p|^{\alpha-1}}\right)|p| \int_{0}^{1}|G(x, y, p)| d y
$$




$$
\begin{aligned}
& \times\left\|\nu_{k}\right\|_{C[0,1]}\left[\frac{1}{|p|^{3-\alpha}}|p| \max _{0 \leq y \leq 1}\left|\widetilde{B}^{0}(y, p)\right|+\frac{\|\varphi\|_{C[0,1]}}{|p|^{2-\alpha}}\right] \\
& +\sum_{k=N_{1}+1}^{N}\left(\left|c_{k}\right|+\frac{|p|^{\alpha}\left|V_{k}(p)\right|}{|p|^{\alpha-1}}\right) \sqrt{|p|} \\
& \times \int_{0}^{1}\left|G_{y}(x, y, p)\right| d y\left\|\mu_{k-N_{1}}\right\|_{C[0,1]} \frac{\left\|\varphi^{\prime}\right\|_{C[0,1]}}{|p|^{\frac{3}{2}-\alpha}}+|p|^{\alpha}\left|\widehat{\Phi}^{0}(x, p)\right| .
\end{aligned}
$$

Note that (6.1) implies $\widehat{\Phi}^{0} \in \mathcal{B}_{\alpha, \sigma_{0}}$ for the function $\widehat{\Phi}^{0}$ defined in (4.22). Using this relation, the assertions (5.3), (5.5) of Lemma 1 and the definitions of the norms $\|\cdot\|_{\gamma, \sigma},\|\cdot\|_{\sigma}$ we obtain from (8.1)

$$
\begin{gathered}
|p|^{\alpha}\left|b^{0}[Z](x, p)\right| \leq \sum_{k=1}^{N_{1}}\left(\left|c_{k}\right|+\frac{\left\|V_{k}\right\|_{\alpha, \sigma}}{|p|^{\alpha-1}}\right) K_{1}\left\|\nu_{k}\right\|_{C[0,1]}\left[\frac{\left\|\widetilde{B}^{0}\right\|_{1, \sigma}}{|p|^{3-\alpha}}+\frac{\|\varphi\|_{C[0,1]}}{|p|^{2-\alpha}}\right] \\
+\sum_{k=N_{1}+1}^{N}\left(\left|c_{k}\right|+\frac{\left\|V_{k}\right\|_{\alpha, \sigma}}{|p|^{\alpha-1}}\right) K_{3}\left\|\mu_{k-N_{1}}\right\|_{C[0,1]} \frac{\left\|\varphi^{\prime}\right\|_{C[0,1]}}{|p|^{\frac{3}{2}-\alpha}}+\left\|\widehat{\Phi}^{0}\right\|_{\alpha, \sigma_{0}}
\end{gathered}
$$

for $\operatorname{Re} p>\sigma, \sigma \geq \sigma_{0}, x \in[0,1]$. Taking here the supremum over $\operatorname{Re} p>\sigma, x \in$ $[0,1]$ and observing the relation $|p|^{\gamma}>\sigma^{\gamma}$ for $\operatorname{Re} p>\sigma$, which holds in the cases $\gamma=\alpha-1,3-\alpha, 2-\alpha, 3 / 2-\alpha$ due to (5.1), we have

$$
\begin{aligned}
\left\|b^{0}[Z]\right\|_{\alpha, \sigma} & \leq \sum_{k=1}^{N_{1}}\left(\left|c_{k}\right|+\frac{\left\|V_{k}\right\|_{\alpha, \sigma}}{\sigma^{\alpha-1}}\right) K_{1}\left\|\nu_{k}\right\|_{C[0,1]}\left[\frac{\left\|\widetilde{B}^{0}\right\|_{1, \sigma}}{\sigma^{3-\alpha}}+\frac{\|\varphi\|_{C[0,1]}}{\sigma^{2-\alpha}}\right] \\
& +\sum_{k=N_{1}+1}^{N}\left(\left|c_{k}\right|+\frac{\left\|V_{k}\right\|_{\alpha, \sigma}}{\sigma^{\alpha-1}}\right) K_{3}\left\|\mu_{k-N_{1}}\right\|_{C[0,1]} \frac{\left\|\varphi^{\prime}\right\|_{C[0,1]}}{\sigma^{\frac{3}{2}-\alpha}}+\left\|\widehat{\Phi}^{0}\right\|_{\alpha, \sigma_{0}}
\end{aligned}
$$

for $\sigma \geq \sigma_{0}$. Finally, observing that $\sigma^{\gamma^{\prime}} \geq \sigma^{\gamma}$ for $\gamma^{\prime}>\gamma$, because $\sigma \geq \sigma_{0} \geq 1$ we arrive at the relation

$$
\left\|b^{0}[Z]\right\|_{\alpha, \sigma} \leq \frac{C_{8}}{\sigma^{\frac{3}{2}-\alpha}}\left(|c|+\frac{\|V\|_{\alpha, \sigma}}{\sigma^{\alpha-1}}\right)+\left\|\widehat{\Phi}^{0}\right\|_{\alpha, \sigma_{0}}, \quad \sigma \geq \sigma_{0}
$$

with a constant $C_{8}$ depending on $K_{1}, K_{3}, \nu, \mu, \widetilde{B}^{0}, \varphi$.

Next, we perform similar transformations with $b^{1}[Z]$ in (4.21) multiplying by $|p|^{\alpha-\frac{1}{2}}$ instead of $|p|^{\alpha}$. We have

$$
\begin{aligned}
& |p|^{\alpha-\frac{1}{2}}\left|b^{1}[Z](x, p)\right| \leq \sum_{k=1}^{N_{1}}\left(\left|c_{k}\right|+\frac{|p|^{\alpha}\left|V_{k}(p)\right|}{|p|^{\alpha-1}}\right) \sqrt{|p|} \int_{0}^{1}\left|G_{x}(x, y, p)\right| d y \\
& \times\left\|\nu_{k}\right\|_{C[0,1]}\left[\frac{1}{|p|^{3-\alpha}}|p| \max _{0 \leq y \leq 1}\left|\widetilde{B}^{0}(y, p)\right|+\frac{\|\varphi\|_{C[0,1]}}{|p|^{2-\alpha}}\right] \\
& +\sum_{k=N_{1}+1}^{N}\left(\left|c_{k}\right|+\frac{|p|^{\alpha}\left|V_{k}(p)\right|}{|p|^{\alpha-1}}\right) \frac{1}{|p|^{\frac{3}{2}-\alpha}}
\end{aligned}
$$




$$
\times\left|\frac{\mu_{k-N_{1}}(x) \varphi^{\prime}(x)}{\lambda(x)}-\int_{0}^{1} G_{x y}(x, y, p) \mu_{k-N_{1}}(y) \varphi^{\prime}(y) d y\right|+|p|^{\alpha-\frac{1}{2}}\left|\widehat{\Phi}^{1}(x, p)\right| .
$$

Note that (6.1) implies $\widehat{\Phi}^{1} \in \mathcal{B}_{\alpha-\frac{1}{2}, \sigma_{0}}$ for the function $\widehat{\Phi}^{1}$ defined in (4.24). Thus, using the assertions (5.4) and (5.6) of Lemma 1 and taking the supremum over $\operatorname{Re} p>\sigma, x \in[0,1]$ we obtain

$$
\begin{array}{r}
\left\|b^{1}[Z]\right\|_{\alpha-\frac{1}{2}, \sigma} \leq \sum_{k=1}^{N_{1}}\left(\left|c_{k}\right|+\frac{\left\|V_{k}\right\|_{\alpha, \sigma}}{\sigma^{\alpha-1}}\right) K_{2}\left\|\nu_{k}\right\|_{C[0,1]} \times\left[\frac{\left\|\widetilde{B}^{0}\right\|_{1, \sigma}}{\sigma^{3-\alpha}}+\frac{\|\varphi\|_{C[0,1]}}{\sigma^{2-\alpha}}\right] \\
+\sum_{k=N_{1}+1}^{N}\left(\left|c_{k}\right|+\frac{\left\|V_{k}\right\|_{\alpha, \sigma}}{\sigma^{\alpha-1}}\right) K_{6}\left\|\mu_{k-N_{1}} \varphi^{\prime}\right\|_{C[0,1]} \frac{1}{\sigma^{\frac{3}{2}-\alpha}}+\left\|\widehat{\Phi}^{1}\right\|_{\alpha-\frac{1}{2}, \sigma_{0}}
\end{array}
$$

for $\sigma \geq \sigma_{0}$. This yields

$$
\left\|b^{1}[Z]\right\|_{1, \sigma} \leq \frac{C_{9}}{\sigma^{\frac{3}{2}-\alpha}}\left(|c|+\frac{\|V\|_{\alpha, \sigma}}{\sigma^{\alpha-1}}\right)+\left\|\widehat{\Phi}^{1}\right\|_{\alpha-\frac{1}{2}, \sigma_{0}}, \quad \sigma \geq \sigma_{0}
$$

with a constant $C_{9}$.

In particular, (8.2) and (8.3) imply $b[Z]=\left(b^{0}[Z], b^{1}[Z]\right) \in \mathcal{B}_{\sigma}$ for $\sigma \geq \sigma_{0}$ and estimate (6.2). To prove (6.3) we denote $Z=Z^{1}-Z^{2}$. Then the components $b^{0}[Z]$ and $b^{1}[Z]$ of the vector $b[Z]=b\left[Z^{1}\right]-b\left[Z^{2}\right]$ are expressed by the formulas (4.20) with $\widehat{\Phi}^{0}=0$ and $(4.21)$ with $\widehat{\Phi}^{1}=0$, respectively. Using the estimates (8.2) and (8.3) for the components of $b[Z]$ and observing that $Z=\frac{c}{p}+V$ with $c=0$ and $V=V^{1}-V^{2}$ we deduce (6.3). The proof is complete.

Proof of the Lemma 4. First we show that the linear operator $A[Z]=$ $\left(A^{0}[Z], A^{1}[Z]\right)$, given by (4.18), (4.19), is bounded in $\mathcal{B}_{\sigma}$ and satisfies estimate (6.4). From (4.18) by $Z=\frac{c}{p}+V$ we get

$$
\begin{aligned}
|p|^{\alpha}\left|\left(A^{0}[Z] B\right)(x, p)\right| & \leq \sum_{k=1}^{N_{1}}\left(\frac{\left|c_{k}\right|}{|p|^{2}}+\frac{|p|^{\alpha}\left|V_{k}(p)\right|}{|p|^{\alpha+1}}+\frac{\left|n_{k}(0)\right|}{|p|}\right) \\
& \times|p| \int_{0}^{1}|G(x, y, p)| d y\left\|\nu_{k}\right\|_{C[0,1]}|p|^{\alpha} \max _{0 \leq y \leq 1}\left|\widehat{B}^{0}(y, p)\right| \\
& +\sum_{k=N_{1}+1}^{N}\left(\frac{\left|c_{k}\right|}{|p|}+\frac{|p|^{\alpha}\left|V_{k}(p)\right|}{|p|^{\alpha+1}}\right) \sqrt{|p|} \int_{0}^{1}\left|G_{y}(x, y, p)\right| d y \\
& \times\left\|\mu_{k-N_{1}}\right\|_{C[0,1]}|p|^{\alpha-\frac{1}{2}} \max _{0 \leq y \leq 1}\left|B^{1}(y, p)\right| .
\end{aligned}
$$

Using Lemma 1 and taking the supremum over $\operatorname{Re} p>\sigma, x \in[0,1]$ we deduce 


$$
\begin{aligned}
\left\|A^{0}[Z] B\right\|_{\alpha, \sigma} & \leq \sum_{k=1}^{N_{1}}\left(\frac{\left|c_{k}\right|}{\sigma^{2}}+\frac{\left\|V_{k}\right\|_{\alpha, \sigma}}{\sigma^{\alpha+1}}+\frac{\left|n_{k}(0)\right|}{\sigma}\right) K_{1}\left\|\nu_{k}\right\|_{C[0,1]}\left\|\widehat{B}^{0}\right\|_{\alpha, \sigma} \\
& +\sum_{k=N_{1}+1}^{N}\left(\frac{\left|c_{k}\right|}{\sigma}+\frac{\left\|V_{k}\right\|_{\alpha, \sigma}}{\sigma^{\alpha}}\right) K_{3}\left\|\mu_{k-N_{1}}\right\|_{C[0,1]}\left\|B^{1}\right\|_{\alpha-\frac{1}{2}, \sigma}
\end{aligned}
$$

for $\sigma \geq \sigma_{0}$. This due to the relations $\sigma^{2} \geq \sigma$ and $\sigma^{\alpha+1} \geq \sigma^{\alpha}$ holding for $\sigma \geq \sigma_{0} \geq 1$ implies

$$
\left\|A^{0}[Z] B\right\|_{\alpha, \sigma} \leq C_{10}\left[\frac{|n(0)|+|c|}{\sigma}+\frac{\|V\|_{\alpha, \sigma}}{\sigma^{\alpha}}\right]\|B\|_{\sigma}, \quad \sigma \geq \sigma_{0},
$$

where $C_{10}$ is a constant. Further, from (4.19) we derive

$$
\begin{aligned}
|p|^{\alpha-\frac{1}{2}} \mid\left(A^{1}[Z] B\right) & (x, p) \mid \leq \sum_{k=1}^{N_{1}}\left(\frac{\left|c_{k}\right|}{|p|^{2}}+\frac{|p|^{\alpha}\left|V_{k}(p)\right|}{|p|^{\alpha+1}}+\frac{\left|n_{k}(0)\right|}{|p|}\right) \\
& \times \sqrt{|p|} \int_{0}^{1}\left|G_{x}(x, y, p)\right| d y\left\|\nu_{k}\right\|_{C[0,1]}|p|^{\alpha} \max _{0 \leq y \leq 1}\left|\widehat{B}^{0}(y, p)\right| \\
& +\sum_{k=N_{1}+1}^{N}\left(\frac{\left|c_{k}\right|}{|p|}+\frac{|p|^{\alpha}\left|V_{k}(p)\right|}{|p|^{\alpha}}\right)\left\|\mu_{k-N_{1}}\right\|_{C[0,1]} \\
& \times\left[\frac{1}{\lambda_{0}}+\int_{0}^{1}\left|G_{x y}(x, y, p)\right| d y\right]|p|^{\alpha-\frac{1}{2}} \max _{0 \leq y \leq 1}\left|B^{1}(y, p)\right| .
\end{aligned}
$$

Here $\lambda_{0}:=\min _{0 \leq x \leq 1} \lambda(x)>0$ because $\lambda \in C[0,1], \lambda(x)>0$, by assumption. Using Lemma 1 and taking the supremum over $\operatorname{Re} p>\sigma, x \in[0,1]$ we get

$$
\left\|A^{1}[Z] B\right\|_{\alpha-\frac{1}{2}, \sigma} \leq C_{11}\left[\frac{|n(0)|+|c|}{\sigma}+\frac{\|V\|_{\alpha, \sigma}}{\sigma^{\alpha}}\right]\|B\|_{\sigma}, \quad \sigma \geq \sigma_{0}
$$

with a constant $C_{11}$.

Putting estimates (8.4) and (8.5) together we have

$$
\|A[Z] B\|_{\sigma} \leq C_{12}\left[\frac{|n(0)|+|c|}{\sigma}+\frac{\|V\|_{\alpha, \sigma}}{\sigma^{\alpha}}\right]\|B\|_{\sigma}, \quad \sigma \geq \sigma_{0}
$$

with a constant $C_{12}$. Due to this relation $A[Z]$ is bounded in $\mathcal{B}_{\sigma}$ and satisfies estimate (6.4).

It remains to prove (6.5). Denoting $Z=Z^{1}-Z^{2}$ the components $A^{0}[Z]$ and $A^{1}[Z]$ of the vector $A[Z]=A\left[Z^{1}\right]-A\left[Z^{2}\right]$ are expressed by the formulas (4.18) and (4.19), respectively, containing $n_{k}(0)=0$. Using the estimate (8.6) for $A[Z]$ and observing that $Z=\frac{c}{p}+V$ with $c=0$ and $V=V^{1}-V^{2}$ we deduce (6.5). Lemma is proved.

Acknowledgement The financial support of Estonian Science Foundation is gratefully acknowledged (Grant nr. 6018). 


\section{References}

[1] B. D. Coleman and M. E. Gurtin. Equipresence and constitutive equation for rigid heat conduction. Z. Angew. Math. Phys., 18, 199-208, 1967.

[2] F. Colombo and A. Lorenzi. Identification of time and space dependent relaxation kernels for materials with memory related to cylindrical domains. $J$. Math. Anal. Appl., 213, 32-90, 1997.

[3] G. Doetsch. Einführung in Theorie und Anwendung der Laplace-Transformation. Birkhäuser Verlag, Basel, 1958. (in German)

[4] J. Janno. Determination of a time- and space-dependent heat flux relaxation function by means of a restricted Dirichlet-to-Neumann operator. Math. Meth. Appl. Sci., 27, 1241-1260, 2004.

[5] J. Janno and A. Lorenzi. A parabolic integro-differential identification problem in a barrelled smooth domain. Z. Anal. Anwen., 25, 103-130, 2006.

[6] J. Janno and L. V. Wolfersdorf. An inverse problem for identification of a time- and space-dependent memory kernel of a special kind in heat conduction. Inverse Problems, 15, 1455-1467, 1999.

[7] J. Janno and L. V. Wolfersdorf. Identification of a special class of memory kernels in one-dimensional heat flow. J. Inv. Ill-Posed Problems, 9, 389-411, 2001.

[8] J. Janno and L. V. Wolfersdorf. Identification of memory kernels in onedimensional heat flow with boundary conditions of the third kind. Inverse Problems in Engineering, 9, 179-198, 2001.

[9] A. Lunardi. On the linear equation with fading memory. SIAM J. Math. Anal., 21, 1213-1224, 1970.

[10] J. W. Nunziato. On heat conduction in materials with memory. Quart. Appl. Math., 29, 187-204, 1971.

[11] E. Pais and J. Janno. Identification of two degenerate time- and space-dependent kernels in a parabolic equation. Electron. J. Differ. Eqns., 2005(108), 1-20, 2005.

[12] I. G. Petrowski. Lectures on Partial Differential Equations. Interscience, New York, 1964.

[13] J. Prüß. Evolutionary Integral Equations and Applications. Birkhäuser Verlag, Boston, 1993. 